\title{
Role of scattering-factor anisotropy in electron, positron, and photon holography
}

\author{
S. Y. Tong, C. W. Mok, and Huasheng Wu \\ Department of Physics, The University of Hong Kong, Hong Kong, China \\ L. Z. Xin \\ Department of Physics and Laboratory for Surface Studies, University of Wisconsin-Milwaukee, Milwaukee, Wisconsin 53201
}

(Received 13 March 1998)

\begin{abstract}
We have studied the angular anisotropy in the scattering factor of electrons, positrons, and photons in solids. We show that as a function of angle, the maximum number of dips in the scattering factor's magnitude and jumps of near $\pi$ in its phase are related to the angular momenta of the bound and resonance states of the potential. The effect of the scattering factor's anisotropy on low-energy electron and positron holographic wave-front reconstruction is discussed. Applying the variable-axis small-cone method, a good-quality reconstructed image is only possible within angular regions where the scattering factor is near isotropic. Thus the usable window for low-energy electron wave-front reconstruction is element dependent; the window size decreases as the atomic number increases. Positrons, on the other hand, are like photons and are not bound by the potential. For positrons or photons, there is no elemental dependence of the usable window and the entire backscattering regime is suitable for holographic reconstruction. We have established two rules that predict the maximum number of magnitude dips and phase jumps in the scattering factor for any element.
\end{abstract}

[S0163-1829(98)06639-9]

\section{INTRODUCTION}

Recently, we have witnessed considerable interest in applying the holographic wave-front reconstruction method to strongly scattering systems. ${ }^{1-23}$ Using the analogy with optical holography, it has been pointed out that in the case of electron scattering in solids, the relative phase between a reference wave and an object wave scattered from an atom $\mathbf{R}_{1}$ from the reference atom is given by $e^{i\left(k R_{1}-\mathbf{k} \cdot \mathbf{R}_{1}\right)} e^{i\left[\phi_{\mathrm{MS}}(\mathbf{k})+\phi_{\mathrm{SW}}(\mathbf{k})+\phi_{\mathrm{SF}}(\mathbf{k})\right]} \cdot{ }^{1,2}$ Here $\mathbf{k}$ is the wave vector of the electron pointing from the reference atom to the detector and $\phi_{\mathrm{MS}}(\mathbf{k}), \phi_{\mathrm{SW}}(\mathbf{k})$, and $\phi_{\mathrm{SF}}(\mathbf{k})$ are $\mathbf{k}$-dependent phases arising from multiple scattering, source wave, and scattering factor, respectively. ${ }^{1-3}$ In optical holography, the three phases are either small or isotropic. ${ }^{24}$ In that case, a Fourier transformation over directions with the kernel $e^{i \mathbf{k} \cdot \mathbf{R}}$ at a single energy is sufficient to reconstruct threedimensional images in real space. ${ }^{4,5}$

For strongly scattering systems, Tong and co-workers ${ }^{6,7}$ and Barton and Terminello ${ }^{8}$ have shown that the multiple scattering phase $\phi_{\mathrm{MS}}(\mathbf{k})$ can be eliminated by integrating normalized diffraction spectra over wave numbers with the kernel $e^{-i(k R-\mathbf{k} \cdot \mathbf{R})}$ and summing the integrals over a set of directions. The directional dependence (i.e., anisotropy) in $\phi_{\mathrm{SW}}(\mathbf{k})$ and $\phi_{\mathrm{SF}}(\mathbf{k})$ can be eliminated by applying a variable-axis small-cone method to the normalized diffraction spectra. ${ }^{3,17}$ In this method, the real-space image at each direction is formed by using the energy-dependent diffraction spectra within a small cone whose axis points in the opposite direction. The axis of the cone varies in-synch with the direction of real-space reconstruction. In an earlier paper ${ }^{3}$ we have examined the anisotropy of the source wave. In this paper we shall study the anisotropy of the scattering factor. We shall develop two rules generally applicable to describe the angular anisotropies in electron, positron, and photon scattering factors. We first state these rules.

Rule 1. The maximum number of dips in the scattering factor $|f(\theta)|$ equals the number of zeros in the Legendre polynomial $P_{l_{\min }}(\theta)$, where $l_{\min }$ is the lowest partial wave that is not a bound or resonance state of the ion-core potential.

Rule 2. At each dip of $|f(\theta)|$, its phase $\phi(\theta)$ jumps by nearly $\pi$. The angular window in which $\phi(\theta)$ is near isotropic decreases as $l_{\min }$ increases.

The ion-core potential in a solid is the (muffin-tin) potential $V(r)$ plus the centrifugal potential $l(l+1) \hbar^{2} / 2 m r^{2}$. Another way to state rule 1 is that the maximum number of dips in $|f(\theta)|$ equals the number of different partial waves $s, l$, $p, \ldots$ that are either bound to or in resonance with the ioncore potential. A corollary to rules 1 and 2 is that for either the positron ${ }^{16}$ or photon ${ }^{25}$ scattering factor, the magnitude $|f(\theta)|$ has no dip and its phase $\phi(\theta)$ has no $\pi$ jump. This is because positrons or photons are not bound by the ion-core potential, therefore, $l_{\min }=0$ in each case. We shall derive these rules and illustrate their usefulness in the following sections.

\section{ANGULAR ANISOTROPY IN THE ELECTRON SCATTERING FACTOR}

It is widely known that electron scattering factors are highly anisotropic in both amplitude and phase. However, no work has explained the origin of the angular anisotropy. In Fig. 1 we show polar plots of the amplitude of the electron scattering factor for $\mathrm{Ni}$ at four energies. We see that the angular anisotropy is energy dependent and the amplitude $\left|f_{e}(\theta)\right|$ exhibits a number of cusps. Besides the dependence on energy, the number of cusps in $\left|f_{e}(\theta)\right|$ also depends on the element. For example, Si has two cusps at $100 \mathrm{eV}$, while $\mathrm{W}$ or Au has four cusps at $300 \mathrm{eV}$. Up to now, no rule exists 

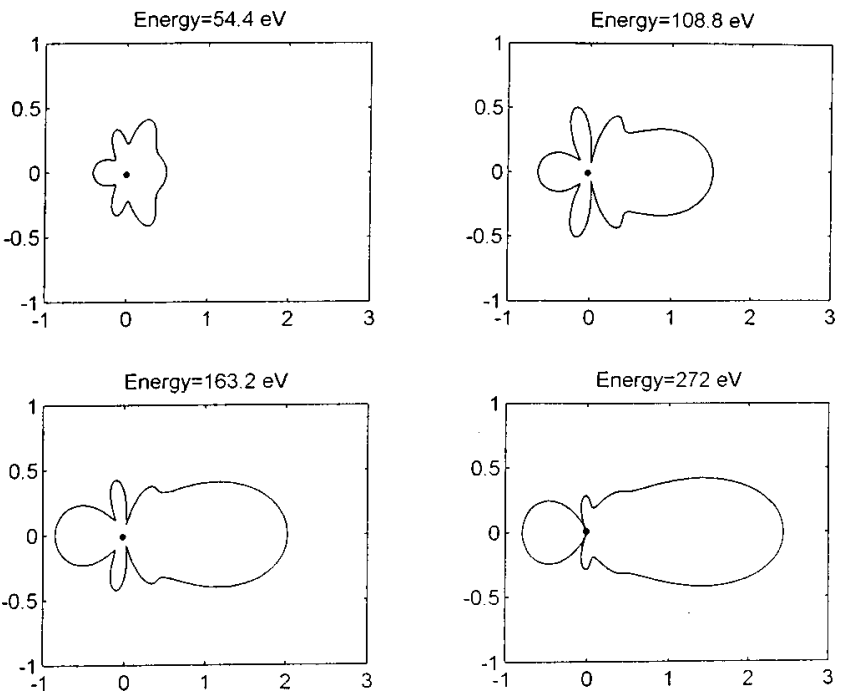

FIG. 1. Polar plots of the amplitude of the Ni electron scattering factor. In each panel, forward scattering is to the right and backscattering to the left. The origin is at $(0,0)$ in each panel.

to predict the maximum number of cusps for a given element. In the following we shall explain the origin of the cusps and establish rules to predict the maximum number for each element. The rules apply to scattering factors of electrons, positrons, and photons.

To begin with, we calculate the electron and positron scattering factors for 12 elemental materials, ranging from rows 2 to 6 of the Periodic Table. For each element, we first obtain the self-consistent full linearized augmented-plane-wave band-structure potential. ${ }^{26}$ We then apply the spherical approximation to this potential (i.e., take its muffin-tin form). From the ion-core potential, we generate the first 30 partial wave phase shifts. While phase shifts are defined up to modulo $\pi$ in the scattering factor, their behavior as $E \rightarrow 0$ depends on how many bound states the potential has. All partial wave phase shifts $\delta_{l}(E \rightarrow 0)=0$ unless the potential has a bound state in that $l$ th subshell. If it does, then $\delta_{l}(E$ $\rightarrow 0$ ) $=n \pi$, where $n$ is the number of bound states with the particular $l$ th quantum number. This is known as Levinson's theorem. $^{27}$ An electron that is not bound by the ion-core potential can be in resonance with the potential. If an electron in an $l$ th subshell is in resonance, the $l$ th partial wave phase shift rises nearly by $\pi$ in a narrow $(1-2 \mathrm{eV})$ energy range. We demonstrate in Figs. 2 and 3 the behavior of the phase shifts at $E$ near zero. For Ni (Fig. 2) and As (Fig. 3), the $l=0$ phase shift starts at $3 \pi$ at $E=0 \mathrm{eV}$ because each element has three $s$-shell bound states. The $l=1$ phase shift starts at $2 \pi$ at $E=0 \mathrm{eV}$ because each element has two $p$-shell bound states. For $\mathrm{Ni}$, the $3 d$ electron is in resonance with the potential; therefore, the $l=2$ phase shift starts at zero and rises rapidly to near $\pi$ at $7 \mathrm{eV}$ and remains near $\pi$ at higher energies. The $3 d$ electron in As is a bound state and hence the $l=2$ phase shifts starts at $\pi$ at $E=0 \mathrm{eV}$. In Table I we list the limiting values of the phase shifts at $E=0 \mathrm{eV}$ for each of the 12 elements. We also indicate the resonance $\pi$ jumps by asterisks. For each element we list the lowest Legendre polynomial for the partial wave that is neither bound to nor in resonance with the ion-core potential. We call this a free state. In Table II we show the electron distribution in the

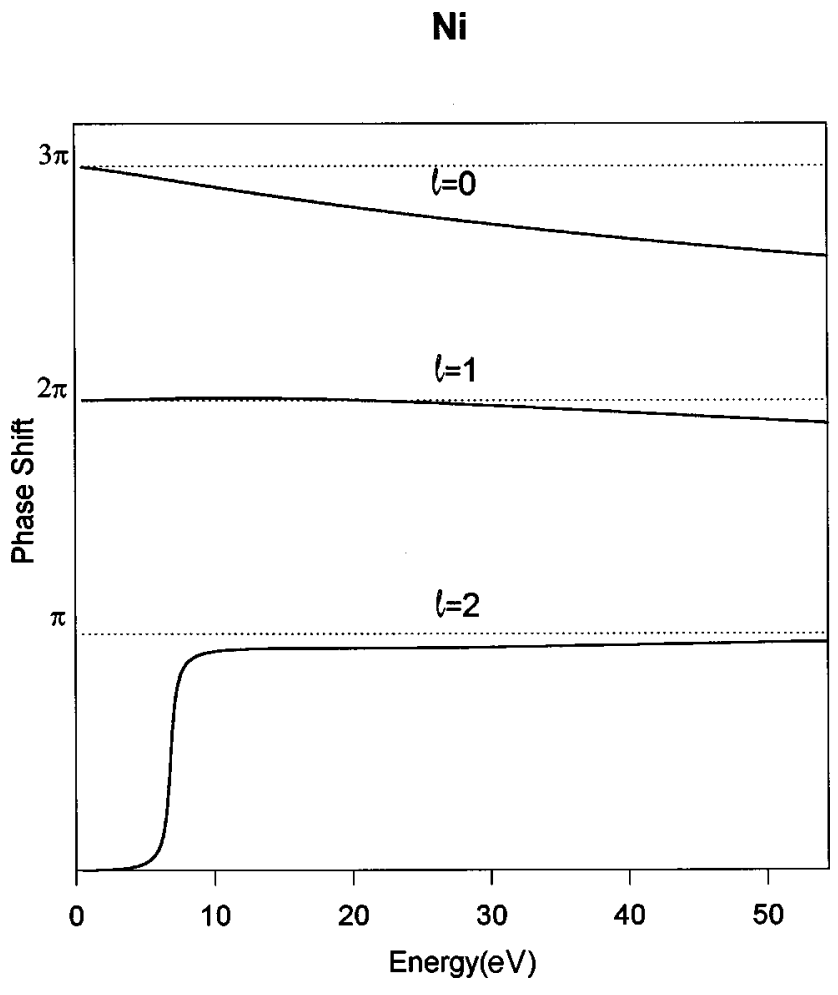

FIG. 2. The $l=0,1$, and 2 phase shifts of $\mathrm{Ni}$ at low energies.

different shells for the 12 elements. The bound and resonance electrons (in asterisks) are shaded.

To save space, we select for presentation here four representative elements for electron scattering, $\mathrm{C}, \mathrm{Si}, \mathrm{Ga}$, and $\mathrm{Pb}$, and two elements for positron scattering, Si and Ga. The interested reader can refer to Ref. 28 for results of the other elements, as well as to obtain the electron and positron phase

\section{As}

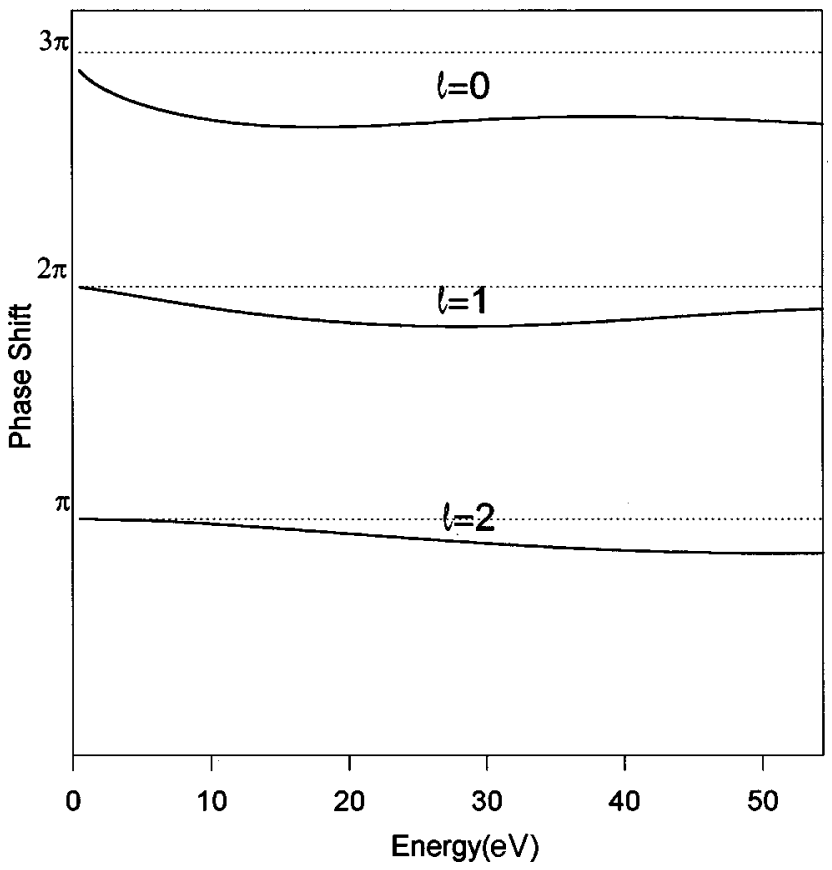

FIG. 3. The $l=0,1$, and 2 phase shifts of As at low energies. 
TABLE I. Limiting values as $E \rightarrow 0$ of each electron partial wave phase shift for the elements listed. $P_{l}$ is the Legendre polynomial of the lowest free-state partial wave. The $\pi$ resonance at low energy is indicated by an asterisk.

\begin{tabular}{ccclcc}
\hline \hline Element & $\begin{array}{c}s \\
(l=0)\end{array}$ & $\begin{array}{c}p \\
(l=1)\end{array}$ & \multicolumn{1}{c}{$\begin{array}{c}d \\
(l=2)\end{array}$} & $\begin{array}{c}f \\
(l=3)\end{array}$ & $\begin{array}{c}g \\
(l=4)\end{array}$ \\
\hline $\mathrm{C}$ & $1 \pi$ & $P_{1}$ & & & \\
$\mathrm{Al}$ & $2 \pi$ & $1 \pi$ & $P_{2}$ & & \\
$\mathrm{Si}$ & $2 \pi$ & $1 \pi$ & $P_{2}$ & & \\
$\mathrm{Fe}$ & $3 \pi$ & $2 \pi$ & $0 \pi+1 \pi^{*}$ & $P_{3}$ & \\
$\mathrm{Ni}$ & $3 \pi$ & $2 \pi$ & $0 \pi+1 \pi^{*}$ & $P_{3}$ & \\
$\mathrm{Cu}$ & $3 \pi$ & $2 \pi$ & $0 \pi+1 \pi^{*}$ & $P_{3}$ & \\
$\mathrm{Ga}$ & $3 \pi$ & $2 \pi$ & $1 \pi$ & $P_{3}$ & \\
$\mathrm{As}$ & $3 \pi$ & $2 \pi$ & $1 \pi$ & $P_{3}$ & \\
$\mathrm{Ag}$ & $4 \pi$ & $3 \pi$ & $1 \pi+1 \pi^{*}$ & $P_{3}$ & \\
$\mathrm{~W}$ & $5 \pi$ & $4 \pi$ & $2 \pi+1 \pi^{*}$ & $1 \pi$ & $P_{4}$ \\
$\mathrm{Au}$ & $5 \pi$ & $4 \pi$ & $2 \pi+1 \pi^{*}$ & $1 \pi$ & $P_{4}$ \\
$\mathrm{~Pb}$ & $5 \pi$ & $4 \pi$ & $3 \pi$ & $1 \pi$ & $P_{4}$ \\
\hline \hline
\end{tabular}

shifts. We show in Fig. 4 the electron scattering factor $\left|f_{e}(\theta)\right|$ for $\mathrm{C}, \mathrm{Si}$, and $\mathrm{Ga}$ at $100 \mathrm{eV}$ and $\mathrm{Pb}$ at $300 \mathrm{eV}$. For anisotropy in the amplitude, we see that the $\mathrm{C}$ scattering factor has a single dip, at $\theta=90^{\circ}$; $\mathrm{Si}$ has two dips, at $\theta$ $=41^{\circ}$ and $110^{\circ}$; Ga has three dips, and $\mathrm{Pb}$ has four dips. While the number of dips and the angles at which they occur are energy dependent, Fig. 4 shows the maximum number of dips for each element. In Fig. 5 we plot the phase $\phi_{e}(\theta)$ for the same four elements. We notice that $\phi_{e}(\theta)$ jumps nearly by $\pi$ at the angle of each dip. To explain these observations, we look at the zeros of the Legendre polynomials. In Fig. 6 we plot the absolute value $\left|P_{l}(\theta)\right|$ for $l=1,2,3$, and 4 . The value of $P_{l}(\theta)$ changes sign before and after each zero. Immediately, we see the correlation between the dips in $\left|f_{e}(\theta)\right|$ and the zeros of $\left|P_{l}(\theta)\right|$ listed in Table I. For example, $\mathrm{C}$ has

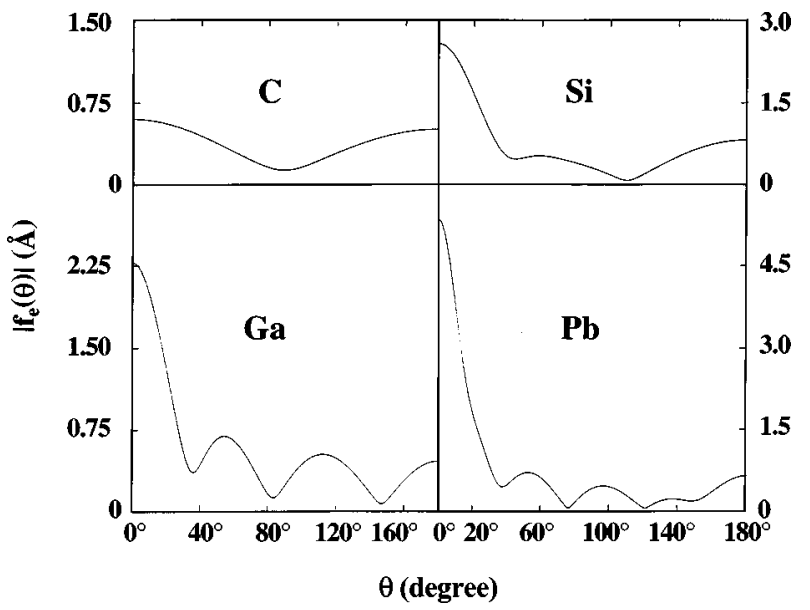

FIG. 4. Magnitude of electron scattering factor vs angle for C, $\mathrm{Si}$, and $\mathrm{Ga}$ at $100 \mathrm{eV}$ and $\mathrm{Pb}$ at $300 \mathrm{eV}$. Left, vertical scale for $\mathrm{C}$, $\mathrm{Ga}$; right, vertical scale for $\mathrm{Si}, \mathrm{Pb}$.

one dip in $\left|f_{e}(\theta)\right|$, while its lowest free-state partial wave $\left|P_{1}(\theta)\right|$ has a single zero. A similar correspondence can be found for the other elements. Even the angles at which $\left|f_{e}(\theta)\right|$ has dips match somewhat closely with the zeros of $\left|P_{l_{\text {min }}}(\theta)\right|$, although we do not expect quantitative correlation because the former is energy dependent.

According to rules 1 and 2, the dips in $\left|f_{e}(\theta)\right|$ and jumps in $\phi_{e}(\theta)$ have origins in the bound and resonant states of the ion-core potential. To understand this, we consider the simplest case, which is $\mathrm{C}$. The $\mathrm{C}$ potential has a $1 \mathrm{~s}$ bound state, thus $\delta_{0}(E)$ starts from $\pi$ at $E=0$. For a weakly bound $1 s$ electron, $\delta_{0}(E)$ decreases from $\pi$ as $E$ increases. The first free-state partial wave $\delta_{1}(E)$, on the other hand, starts from zero at $E=0$ and rises rapidly through $\pi / 2$, peaks below $\pi$, and then slowly decreases at higher energies. Thus, at 100 $\mathrm{eV}$, the angular anisotropy of the scattering factor is dominated by that of the first free-state partial wave, which behaves as $P_{1}(\theta)$ (see Fig. 7). The dominance of the $l=1$

TABLE II. Electrons in different shells for each of the 12 elements. The bound or resonance electrons (denoted by asterisks) are shaded.

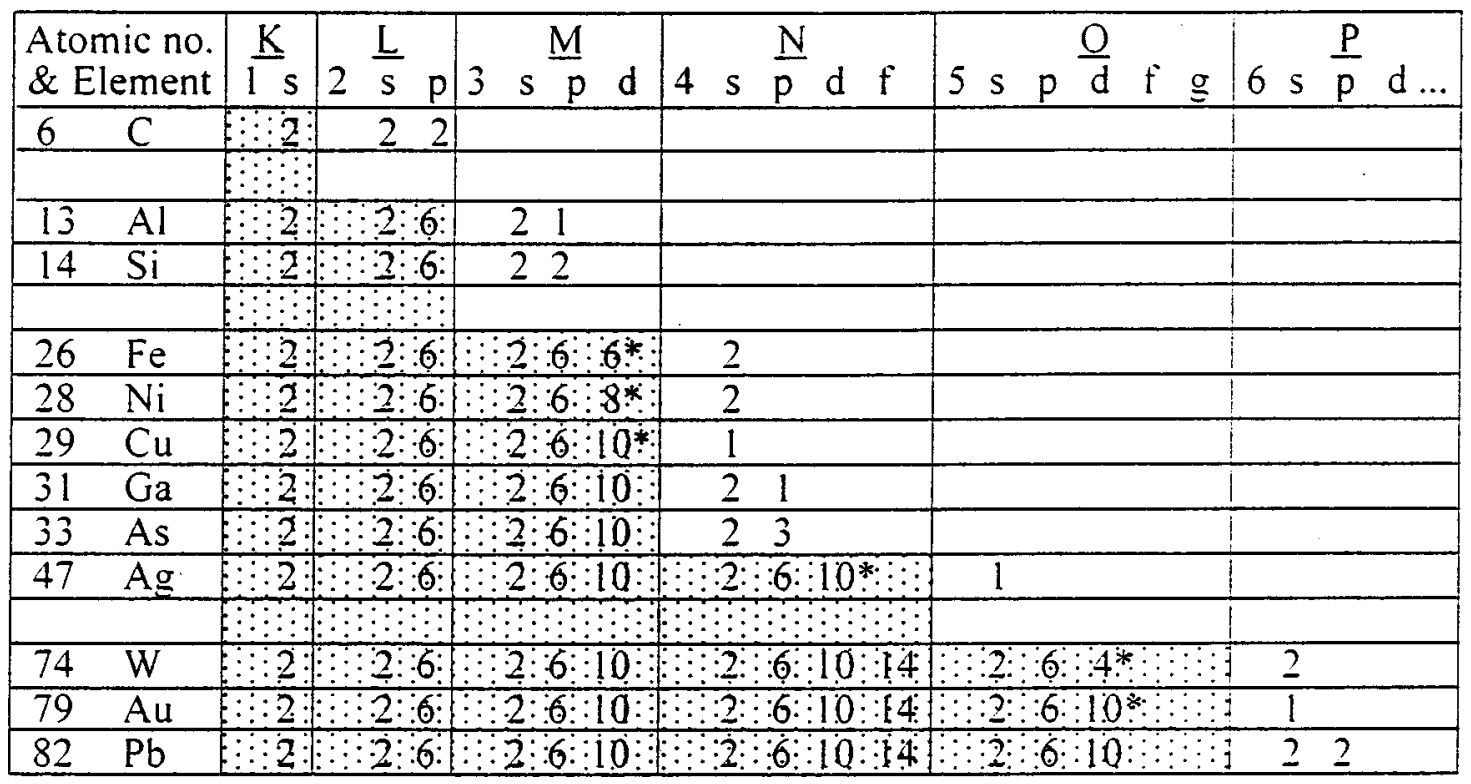




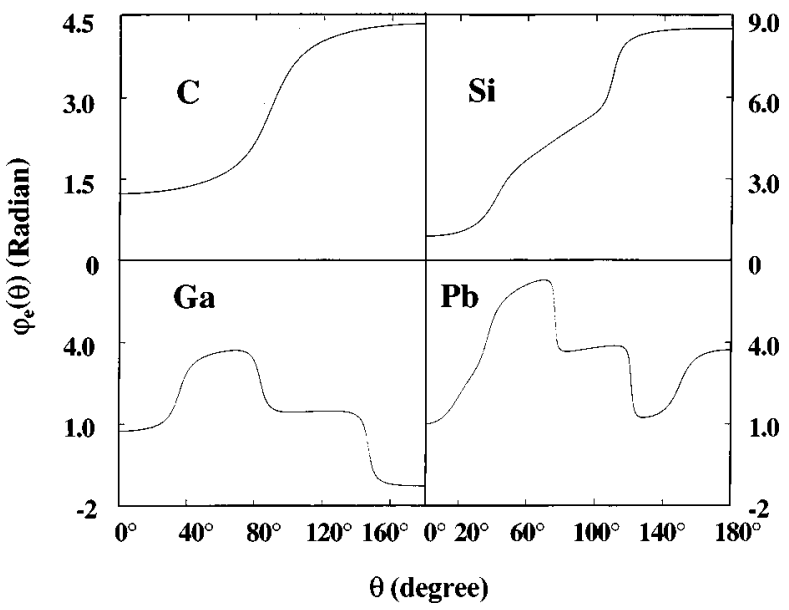

FIG. 5. Phase of electron scattering factor vs angle for $\mathrm{C}$, $\mathrm{Si}$, and $\mathrm{Ga}$ at $100 \mathrm{eV}$ and $\mathrm{Pb}$ at $300 \mathrm{eV}$.

partial wave over the $l=0$ partial wave is accentuated by the $2 l+1$ factor in the scattering factor. Since $P_{1}(\theta)$ has a zero at $90^{\circ}$ and its sign changes at that angle, the amplitude of carbon's scattering factor goes through a minimum near that angle and its phase changes by $\pi$. The latter is because as $P_{1}(\theta)$ changes sign, both the real and imaginary parts of the scattering factor also change sign $\left[f_{l}(\theta)=(2 l\right.$ $\left.+1) \sin ^{2} \delta_{l} P_{l}(\theta)+i(2 l+1) \cos \delta_{l} \sin \delta_{l} P_{l}(\theta)\right]$. Here $f_{l}(\theta)$ is the $l$ th partial wave component of the scattering factor $f(\theta)$.

Turning now to $\mathrm{Si}$, Table I shows that at $E=0, \delta_{0}$ starts at $2 \pi$ and $\delta_{1}$ starts at $\pi$. The two phase shifts decrease in value as $E$ gets larger. The first free-state partial wave $l=2$ again rises rapidly through $\pi / 2$. Because of the $2 l+1$ factor, it is not necessary to consider the effect of $l=0$ relative to $l$ $=2$. At $E=100 \mathrm{eV}$ for $\mathrm{Si}, \delta_{2}$ is near $\pi / 2$, while $\delta_{1}$ is crossing the abscissa. Thus the angular anisotropy of the Si scattering factor at $100 \mathrm{eV}$ is dominated by the free-state $l=2$ partial wave. From Fig. 7 it is easy to see that the situation with the Ga scattering factor is similarly dominated by the angular anisotropy of the $l=3$ partial wave. The $l=2$ phase shift starts at $\pi$ and remains close to this value over a few hundred $\mathrm{eV}$. For the transitional metal elements $\mathrm{Fe}, \mathrm{Ni}$, and $\mathrm{Cu}$, the $l=2$ phase shift "resonates" from zero to near $\pi$ at a very low energy and remains close to $\pi$ over a few hundred

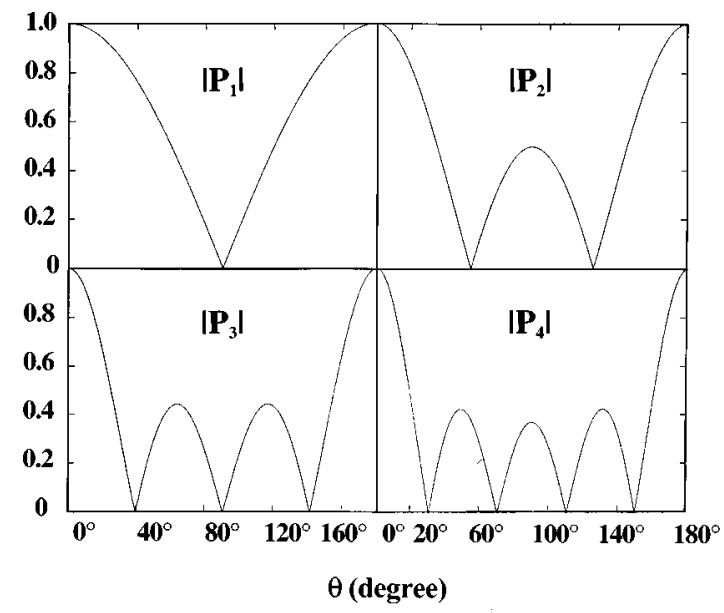

FIG. 6. Absolute value of Legendre polynomials vs angle.
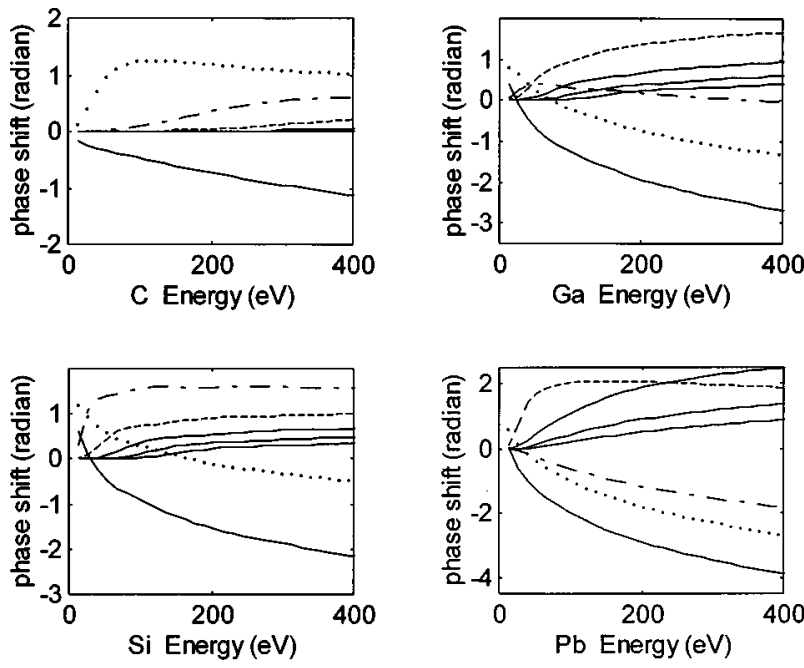

FIG. 7. First few electron partial wave phase shifts for C, Si, Ga, and $\mathrm{Pb}$. Negative slope solid line $(l=0)$, dotted line $(l=1)$, dashdotted line $(l=2)$, and dashed line $(l=3)$. Positive slope solid lines $(l=4,5, \ldots)$. The phase shifts at $E=0 \mathrm{eV}$ are expressed in radians, modulo $\pi$.

$\mathrm{eV}$. In all these materials ( $\mathrm{Fe}, \mathrm{Ni}, \mathrm{Cu}, \mathrm{Ga}, \mathrm{As}$, and $\mathrm{Ag})$, the scattering factor is dominated by the angular anisotropy of the $l=3$ free-state partial wave.

The situation with the row six heavy metals $(\mathrm{W}, \mathrm{Au}$, and $\mathrm{Pb}$ ) is only slightly more complicated. Here the $4 f$ core level is more tightly bound, resulting in $\delta_{3}$, which starts from $\pi$ at $E=0$ to increase through another $\pi / 2$, before slowly decreasing at higher energies. The first free-state partial wave $\delta_{4}$ starts from 0 and increases through $\pi / 2$ as $E$ increases. We can see from Fig. 7 that for $\mathrm{Pb}$, the anisotropy of the scattering factor reflects those of $P_{4}$ at $300 \mathrm{eV}$ and those of $P_{3}$ at $100 \mathrm{eV}$. The same holds true for $\mathrm{W}$ and $\mathrm{Au}$. The reader can refer to Ref. 28 for the angular anisotropies of $\mathrm{W}, \mathrm{Au}$, and $\mathrm{Pb}$ at $100 \mathrm{eV}$. The fact that $\left|f_{e}(\theta)\right|$ of $\mathrm{Pb}$ has four dips at $300 \mathrm{eV}$ and only three at $100 \mathrm{eV}$ does not affect the validity of rule 1 , which is concerned with the maximum number of dips.

In the variable-axis small-cone method of low-energy electron holography, ${ }^{1-3,17}$ we separate out diffraction paths from atoms where the scattering factor is nearly isotropic. To

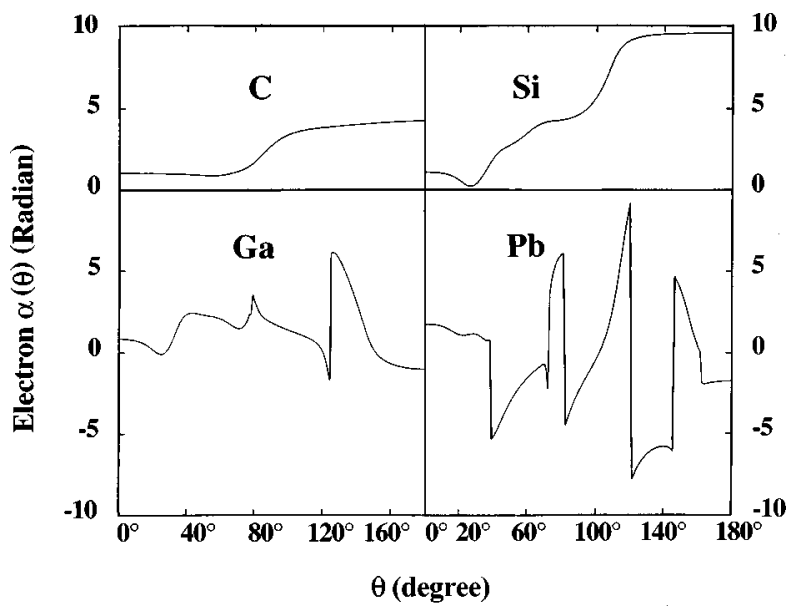

FIG. 8. Coefficient $\alpha(\theta)$ vs electron scattering angle obtained for $\mathrm{C}, \mathrm{Si}, \mathrm{Ga}$, and $\mathrm{Pb}$ 


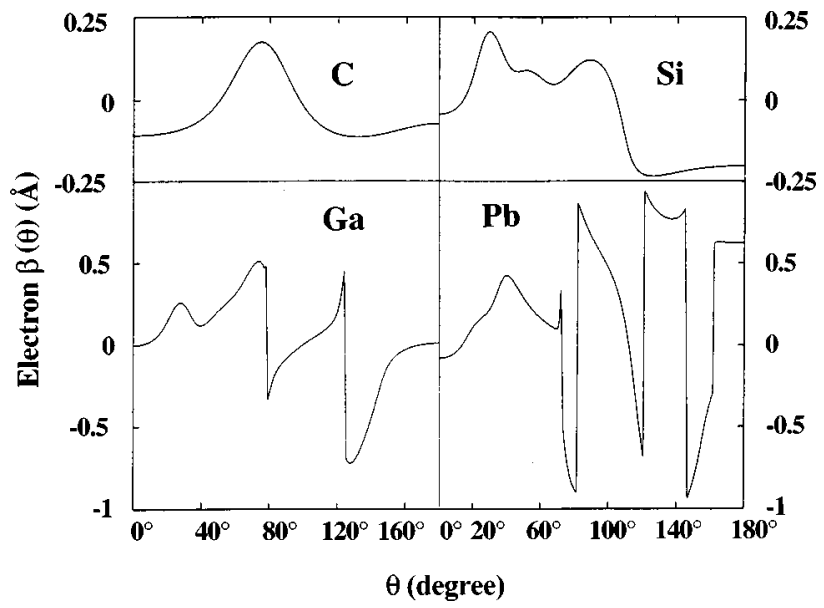

FIG. 9. Coefficient $\beta(\theta)$ vs electron scattering angle obtained for $\mathrm{C}, \mathrm{Si}, \mathrm{Ga}$, and $\mathrm{Pb}$.

understand how the angular anisotropy in the phase of the scattering factor affects the small-cone window, we follow previous works ${ }^{1-3,6}$ and expand the phase as

$$
\phi_{k}(\theta)=\alpha(\theta)+\beta(\theta) k+\cdots .
$$

We have evaluated the coefficients $\alpha(\theta)$ and $\beta(\theta)$, which are averaged quantities, over an energy range considered. We show in Figs. 8 and 9 the two coefficients, respectively, for electron scattering of $\mathrm{C}, \mathrm{Si}, \mathrm{Ga}$, and $\mathrm{Pb}$. The energy range used for $\mathrm{C}$ is $4-12$ hartrees $(109-326 \mathrm{eV})$, while that for $\mathrm{Si}$, $\mathrm{Ga}$, and $\mathrm{Pb}$ is $4-15$ hartrees $(109-408 \mathrm{eV})$. The holographic method requires that $\alpha(\theta)$ and $\beta(\theta)$ be nearly isotropic within the angular cone of wave-front reconstruction. ${ }^{1-3}$ We see from the figures that in the backscattering regime, this condition is satisfied for angular cones with half angles of $80^{\circ}$ for $\mathrm{C}, 60^{\circ}$ for $\mathrm{Si}, 30^{\circ}$ for $\mathrm{Ga}$, and $15^{\circ}$ for $\mathrm{Pb}$. The reason why we consider only the backscattering regime is because the holographic method also requires the magnitude $|f(\theta)|$ to be near isotropic. This condition eliminates the forwardscattering region where $|f(\theta)|$ is strongly peaked. Within the respective backscattering small cones, $\alpha(\theta)$ varies by less than $1 \mathrm{rad}$ and $\beta(\theta)$ varies by less than $0.05 \AA$. The coeffi-

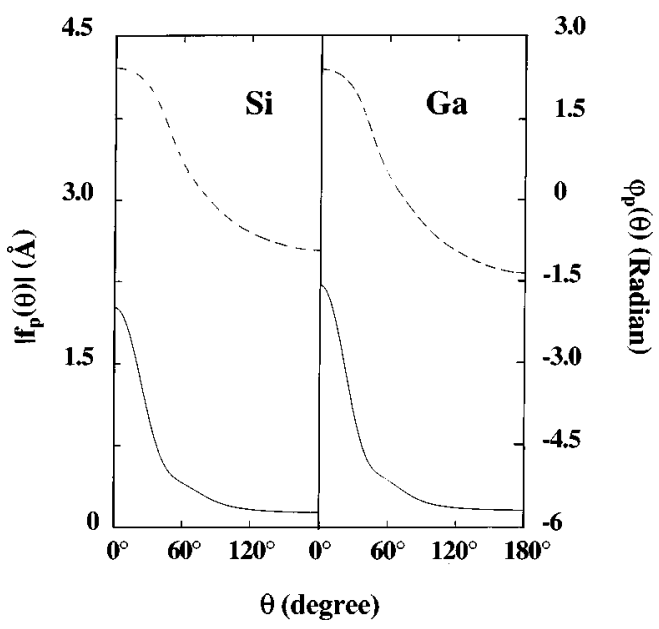

FIG. 10. Magnitude (solid line) and phase (dashed line) of the positron scattering factor for $\mathrm{Si}$ and $\mathrm{Ga}$ at $100 \mathrm{eV}$. Vertical scale: left, magnitude; right, phase.

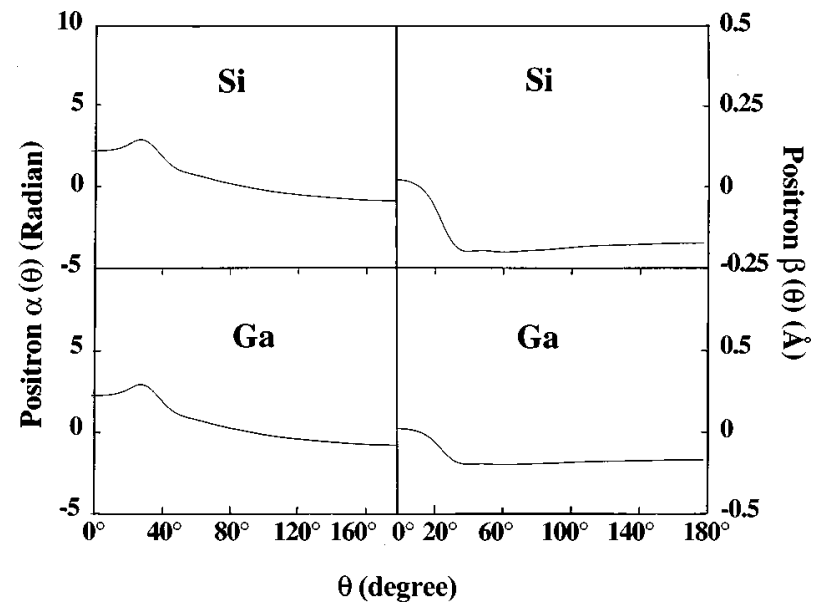

FIG. 11. Positron's coefficients $\alpha(\theta)$ and $\beta(\theta)$ vs scattering angle for $\mathrm{Si}$ and $\mathrm{Ga}$.

cient $\beta(\theta)$ produces an error in the position of the reconstructed image and a small $\beta(\theta)$ guarantees that this error would be small (see Ref. 2 for an explicit formula of the error). The angular window affects the radial and transverse resolutions of the reconstructed image. Reference 2 gives the dependence of image resolution on the angular window.

\section{ISOTROPIC BEHAVIOR OF THE POSITRON BACKSCATTERING FACTOR}

Because positrons and photons are not bound by the atomic potential, it follows from rules 1 and 2 that (i) $\left|f_{\mathrm{ph}}(\theta)\right|$ and $\left|f_{p}(\theta)\right|$ have no dip, i.e., they are monotonically decreasing functions of $\theta$, and (ii) the phases of $f_{\mathrm{ph}}(\theta)$ and $f_{p}(\theta)$ do not have sharp jumps. We show in Fig. 10 the positron's $\left|f_{p}(\theta)\right|$ and phase $\phi_{p}(\theta)$ for $\mathrm{Si}$ and $\mathrm{Ga}$ at $100 \mathrm{eV}$. In the backscattering regime, $\left|f_{p}(\theta)\right|$ is nearly isotropic; hence it satisfies the requirement of the holographic reconstruction method. The coefficients $\alpha(\theta)$ and $\beta(\theta)$ for the positron's scattering phase $\phi_{p}(\theta)$ evaluated in the energy range 4-15 hartrees are shown in Fig. 11. Since $\alpha(\theta)$ and $\beta(\theta)$ are nearly isotropic in the scattering range $50^{\circ}-180^{\circ}$, there is no need to apply a small cone to the wave-front reconstruction. The larger usable angular range for positron holography means that the reconstructed image will have a better transverse resolution. ${ }^{2}$ In Fig. 12 we show the first few phase shifts of positron scattering for $\mathrm{Si}$ and $\mathrm{Ga}$. Because no core level is present, all the phase shifts start from zero at $E=0$. Another consequence of the repulsive potential is that all positron phase shifts are negative. ${ }^{29}$

Finally, in Figs. 13 and 14 we compare the magnitudes of
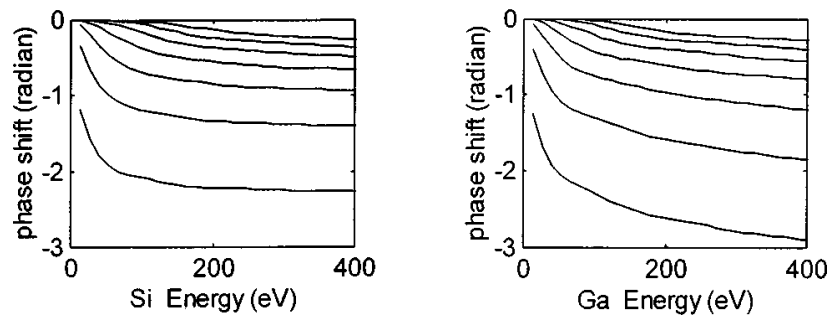

FIG. 12. First few positron phase shifts for $\mathrm{Si}$ and Ga. $l$ $=0,1,3, \ldots$. 


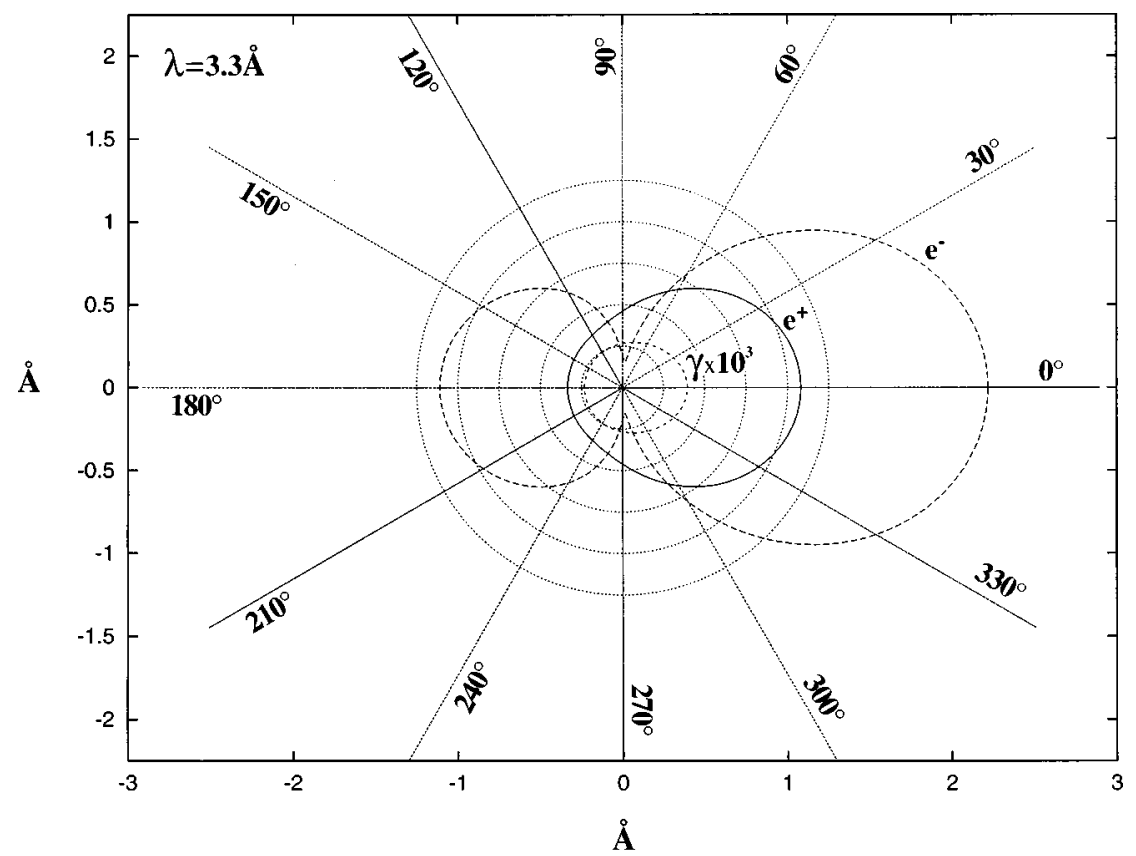

FIG. 13. Radial plot of $|f(\theta)|$ for the electron $\left(e^{-}\right)$, positron $\left(e^{+}\right)$, and photon $(\gamma)$ at a wavelength of $3.3 \AA$. For the radial scale, each circular ring is $0.25 \AA$. The scattering factor of the photon is multiplied by $10^{3}$.

the $\mathrm{Si}$ scattering factor for electrons $\left(e^{-}\right)$, positrons $\left(e^{+}\right)$, and photons $(\gamma)$ at $3.3-\AA$ and $1.3-\AA$ wavelengths, respectively. The $3.3-\AA$ wavelength corresponds to an $e^{+}$or $e^{-}$ energy of $13.6 \mathrm{eV}$ and a $\gamma$ energy of $3.8 \mathrm{keV}$. The $1.3-\AA$ wavelength corresponds to an $e^{+}$or $e^{-}$energy of $95 \mathrm{eV}$ and a $\gamma$ energy of $9.6 \mathrm{keV}$. From these figures we see that $\left|f_{p}(\theta)\right|$ and $f_{\mathrm{ph}}(\theta) \mid$ are nearly isotropic in the entire backscattering regime. Moreover, these scattering factors are smooth functions at all angles. The electron scattering factor, on the other hand, contains large angular anisotropies, which must be avoided in holographic wave-front reconstruction.

\section{COMPARING MULTIPLE SCATTERING IN ELECTRON, POSITRON, AND PHOTON DIFFRACTION}

In this section we provide a simple formula for estimating the importance of multiple scattering for a particle in a solid. We can use the scattering factors shown in Figs. 13 and 14 to estimate the size of multiple scatterings. Following previous works, ${ }^{1,2,7}$ the ratio of a double-scattering event vs singlescattering event is given, within the small-atom approximation, by

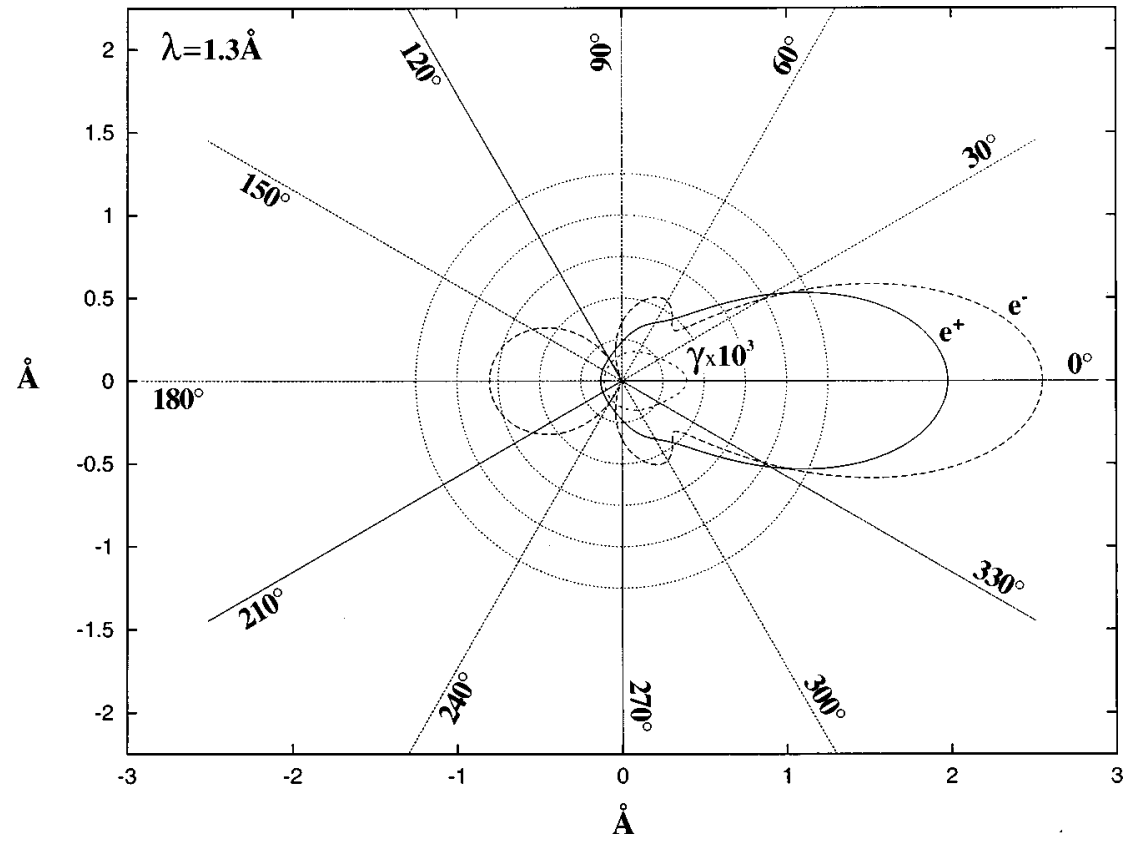

FIG. 14. Same as in Fig. 13, except for a 1.3-Å wavelength. 

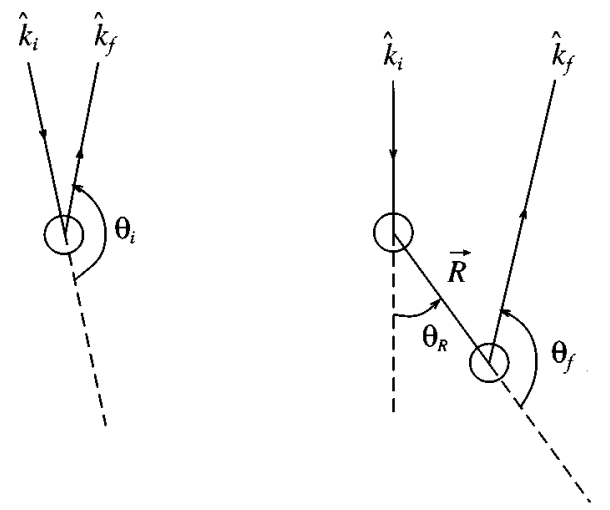

FIG. 15. Schematic diagram of single scattering (left) and double scattering (right).

$$
\frac{\mathcal{M}}{\mathcal{S}}=\frac{\left|f\left(\theta_{R}\right)\right|}{R} \frac{\left|f\left(\theta_{f}\right)\right|}{\left|f\left(\theta_{i}\right)\right|},
$$

where $\mathcal{M}$ denotes a double-scattering event and $\mathcal{S}$ a singlescattering event. The angles $\theta_{R}, \theta_{f}$, and $\theta_{i}$ are shown in Fig. 15. If we assume that the near backscattering factors $\left|f\left(\theta_{f}\right)\right| \approx\left|f\left(\theta_{i}\right)\right|$; then we obtain a useful estimate of the size of double scattering

$$
\frac{\mathcal{M}}{\mathcal{S}} \approx \frac{\left|f\left(\theta_{R}\right)\right|}{R} .
$$

In other words, the ratio of $\mathcal{M}$ to $\mathcal{S}$ is given by the ratio of the scattering factor in direction $\theta_{R}$ to the bond length. For $\mathrm{Si}$, the nearest-neighbor bond length is $2.35 \AA$. From Fig. 14 the positron scattering factor in the forward zone $0^{\circ} \leqslant \theta$ $\leqslant 90^{\circ}$ is larger than $0.25 \AA$. Because Eq. (3) is the ratio of amplitudes, the double-scattering contribution to the intensity is $2 \mathcal{M} / \mathcal{S}$, which gives a value of the double-scattering contribution for positron scattering in the forward zone greater than $20 \%$. In the backward zone $90^{\circ}<\theta \leqslant 180^{\circ}$, double-scattering contribution for positron scattering is near $10 \%$. In comparison, for electrons, the double-scattering contribution to the forward zone is at least $40 \%$ and it can be as large as $60 \%$ at backward $\pi$ scattering. For photon scattering, the ratio $\left|f\left(\theta_{R}\right)\right| / R \ll 1$, indicating that single scattering is sufficient.

\section{CONCLUSIONS}

We have shown that for electron scattering, the angular anisotropy in the scattering factor is caused by bound and resonance states of the atomic potential. These states cause the first few partial wave phase shifts to be near $n \pi$ at low energy. This fact, coupled with the $2 l+1$ factor, results in the electron scattering factor of an element exhibiting the angular anisotropy of its first free-state partial wave. This behavior allows us to predict the maximum number of dips in $\left|f_{e}(\theta)\right|$.

We have also shown that for positron or photon diffraction, because of the absence of bound or resonance states, the scattering factor is isotropic in the entire backscattering regime. Thus positron and photon diffraction spectra are well suited for holographic wave-front reconstruction and the larger usable angular window produces better resolved images. $^{1-3}$ The reader may be interested to learn that by removing the first few partial waves from a positron's scattering factor, it is possible to create similar angular anisotropies seen for electrons. The removal of the first few phase shifts is equivalent to simulating bound or resonance states for these partial waves. For example, if we remove the $l$ $=0$ and 1 phase shifts from the positron's scattering factor of $\mathrm{Si}$, the remaining (artificial) scattering factor exhibits two dips (as in $P_{2}$ ). Similarly, if we remove the $l=0,1$, and 2 phase shifts, the resulting $\mathrm{Si}$ scattering factor exhibits three dips (as in $P_{3}$ ). The reader can refer to Ref. 28 for these results.

\section{ACKNOWLEDGMENTS}

This work was supported in part by the Hong Kong Research Grant Council, the RGC Central Allocation Vote, HKU CRCG, DOE Grant No. DE-FG02-84ER 45076, and NSF Grant No. DMR-9214054. We also acknowledge helpful discussions with D. R. Snider.
${ }^{1}$ S. Y. Tong, Adv. Phys. (to be published).

${ }^{2}$ S. Y. Tong, T. P. Chu, Huasheng Wu, and H. Huang, Surf. Rev. Lett. 4, 459 (1997).

${ }^{3}$ S. Y. Tong, H. Li, and H. Huang, Phys. Rev. B 51, 1850 (1995).

${ }^{4}$ A. Szoeke, in Short Wavelength Coherent Radiation: Generation and Applications, edited by D. T. Attwood and J. Boker, AIP Conf. Proc. No. 147 (AIP, New York, 1986).

${ }^{5}$ J. J. Barton, Phys. Rev. Lett. 61, 1356 (1988).

${ }^{6}$ S. Y. Tong, H. Li, and H. Huang, Surf. Rev. Lett. 1, 303 (1994).

${ }^{7}$ S. Y. Tong, H. Huang, and C. M. Wei, Phys. Rev. B 46, 2452 (1992).

${ }^{8}$ J. J. Barton, Phys. Rev. Lett. 67, 3106 (1991); J. J. Barton and L. J. Terminello, in The Structure of Surfaces III, edited by S. Y. Tong, M. A. Van Hove, K. Takayanagi, and X. D. Xie (Springer, Berlin, 1991).

${ }^{9}$ S. Y. Tong, H. Li, and H. Huang, Phys. Rev. B 46, 4155 (1992).

${ }^{10}$ C. M. Wei and S. Y. Tong, Surf. Sci. Lett. 274, L577 (1992).
${ }^{11}$ J. G. Tobin, G. D. Waddill, H. Li, and S. Y. Tong, Phys. Rev. Lett. 70, 4150 (1993).

${ }^{12}$ H. Wu, G. J. Lapeyre, H. Huang, and S. Y. Tong, Phys. Rev. Lett. 71, 251 (1993).

${ }^{13}$ C. M. Wei, S. Y. Tong, H. Wedler, M. A. Mendez, and K. Heinz, Phys. Rev. Lett. 72, 2434 (1994).

${ }^{14}$ C. M. Wei, I. H. Hong, P. R. Jeng, S. C. Shyu, and Y. C. Chou, Phys. Rev. B 49, 5109 (1994).

${ }^{15}$ I. H. Hong, P. R. Jeng, S. C. Shyu, Y. C. Chou, and C. M. Wei, Surf. Sci. Lett. 312, L743 (1994).

${ }^{16}$ S. Y. Tong, H. Huang, and X. Q. Guo, Phys. Rev. Lett. 69, 3654 (1992).

${ }^{17}$ Huasheng Wu and G. J. Lapeyre, Phys. Rev. B 51, 14549 (1995).

${ }^{18}$ M. Zharnikov, M. Weinelt, P. Zebisch, M. Stickler, and H. P. Steinruck, Phys. Rev. Lett. 73, 3548 (1994).

${ }^{19}$ D. K. Saldin, K. Reuter, P. L. De Andres, H. Wedler, X. Chen, J. B. Pendry, and K. Heinz, Phys. Rev. B 54, 8172 (1996). 
${ }^{20}$ S. Y. Tong, C. M. Wei, T. C. Zhao, H. Huang, and H. Li, Phys. Rev. Lett. 66, 60 (1991).

${ }^{21}$ G. R. Harp, D. K. Saldin, and B. P. Tonner, Phys. Rev. Lett. 65, 1012 (1990).

${ }^{22}$ I. H. Hong, D. K. Liao, Y. C. Chou, C. M. Wei, and S. Y. Tong, Phys. Rev. B 54, 4762 (1996).

${ }^{23}$ S. Y. Tong, Hua Li, and H. Huang, Phys. Rev. Lett. 67, 3102 (1991).

${ }^{24}$ D. Gabor, Nature (London) 161, 777 (1948).

${ }^{25}$ T. Gog, P. M. Len, G. Materlik, D. Bahr, C. S. Fadley, and C.
Sanchez-Hanke, Phys. Rev. Lett. 76, 3132 (1996).

${ }^{26}$ E. Wimmer, H. Krakauser, M. Weinert, and A. J. Freeman, Phys. Rev. B 24, 864 (1981); H. L. Jansen and A. J. Freeman, ibid. 30, 561 (1984).

${ }^{27}$ R. G. Newton, Scattering Theory of Waves and Particles (McGraw-Hill, New York, 1966).

${ }^{28}$ C. W. Mok, Huashang Wu, and S. Y. Tong, Surf. Rev. Lett. (to be published).

${ }^{29}$ Hua Li and S. Y. Tong, Surf. Sci. Lett. 281, L347 (1993). 